Journal of

Molecular Microbiology

\title{
Control of Transposon-Mediated Directed Mutation by the Escherichia coli Phosphoenolpyruvate:Sugar Phosphotransferase System
}

\author{
Milton H. Saier Jr. Z Zhongge Zhang \\ Department of Molecular Biology, Division of Biological Sciences, University of California at San Diego, \\ La Jolla, Calif., USA
}

\section{Key Words}

Directed mutation - Transposon - Insertion sequence-5 ·

Cyclic AMP · cAMP receptor protein · GlpR · Inducer previous publications. Direct involvement of the glycerol repressor, GlpR, and the cAMP receptor protein, Crp, in the regulation of transposon-mediated directed mutation has been demonstrated.

(c) 2015 S. Karger AG, Basel

\begin{abstract}
The phosphoenolpyruvate:sugar phosphotransferase system (PTS) has been shown to control transport, cell metabolism and gene expression. We here present results supporting the novel suggestion that in certain instances it also regulates the mutation rate. Directed mutations are defined as mutations that occur at higher frequencies when beneficial than when neutral or detrimental. To date, the occurrence of directed point mutations has not been documented and confirmed, but a few examples of transposon-mediated directed mutations have been reported. Here we focus on the first and best-studied example of directed mutation, which involves the regulation of insertion sequence-5 hopping into a specific site upstream of the glpFK glycerol utilization operon in Escherichia coli. This insertional event specifically activates expression of the glpFK operon, allowing the growth of wild-type cells with glycerol as a carbon source in the presence of nonmetabolizable glucose analogues which normally block glycerol utilization. The sugar-transporting PTS controls this process by regulating levels of cytoplasmic glycerol-3-phosphate and cyclic (c)AMP as established in
\end{abstract}

\section{Introduction}

The Escherichia coli Phosphotransferase System

The bacterial phosphotransferase system (PTS) functions in a variety of regulatory capacities [Barabote and Saier, 2005; Postma et al., 1993]. One of the best-characterized of these is the process by which it regulates inducer uptake and catabolite repression (fig. 1) [Saier, 1989]. Early genetic and physiological evidence supported a mechanism whereby the phosphorylation state of a protein of the PTS, the Enzyme IIA specific for glucose $\left(\right.$ IIA $\left.^{\mathrm{Glc}}\right)$, allosterically inhibits the activities of a number of permeases and catabolic enzymes, the lactose, galactose, melibiose and maltose permeases, as well as glycerol kinase [Osumi and Saier, 1982; Saier, 1993; Saier and Roseman, 1976]. Extensive biochemical evidence as well as high-resolution X-ray crystallographic 3-dimentional structural data now supports this model [Bluschke et al., 2006; Hoischen et al., 1996; Saier et al., 1978; Seok et al.,

\section{KARGER 125}

C 2015 S. Karger AG, Base

$1464-1801 / 15 / 0253-0226 \$ 39.50 / 0$

E-Mail karger@karger.com

www.karger.com/mmb
Milton H. Saier Jr.

Department of Molecular Biology, Division of Biological Sciences

University of California at San Diego

La Jolla, CA 92093-0116 (USA)

E-Mail msaier@ucsd.edu 
Fig. 1. Proposed mechanism for regulation of glycerol kinase (GlpK) and adenylate cyclase (AC) by the PTS in enteric bacteria including E. coli. Enzyme IIA ${ }^{\text {Glc }}$ (IIA ${ }^{\text {Glc }}$ ) is the central regulatory protein that is reversely phosphorylated by the two general energy-coupling proteins of the PTS, Enzyme I (I) and HPr, which are sequentially phosphorylated by phosphoenolpyruvate (PEP). IIA ${ }^{\mathrm{Glc}}$ interacts with the target protein, GlpK or AC. As all of the phosphoproteins of the PTS are high energy, their phosphorylation is reversible. Only phosphorylated IIA ${ }^{\text {Glc }}$ activates adenylate cyclase, and only the free form of IIA ${ }^{\text {Glc }}$ inhibits glycerol kinase and the non-PTS permeases, although some evidence suggests that the free form of IIA ${ }^{\text {Glc }}$ inhibits adenylate cyclase. II represents an Enzyme II specific for a particular sugar (S). Modified from Saier [1989], with permission.

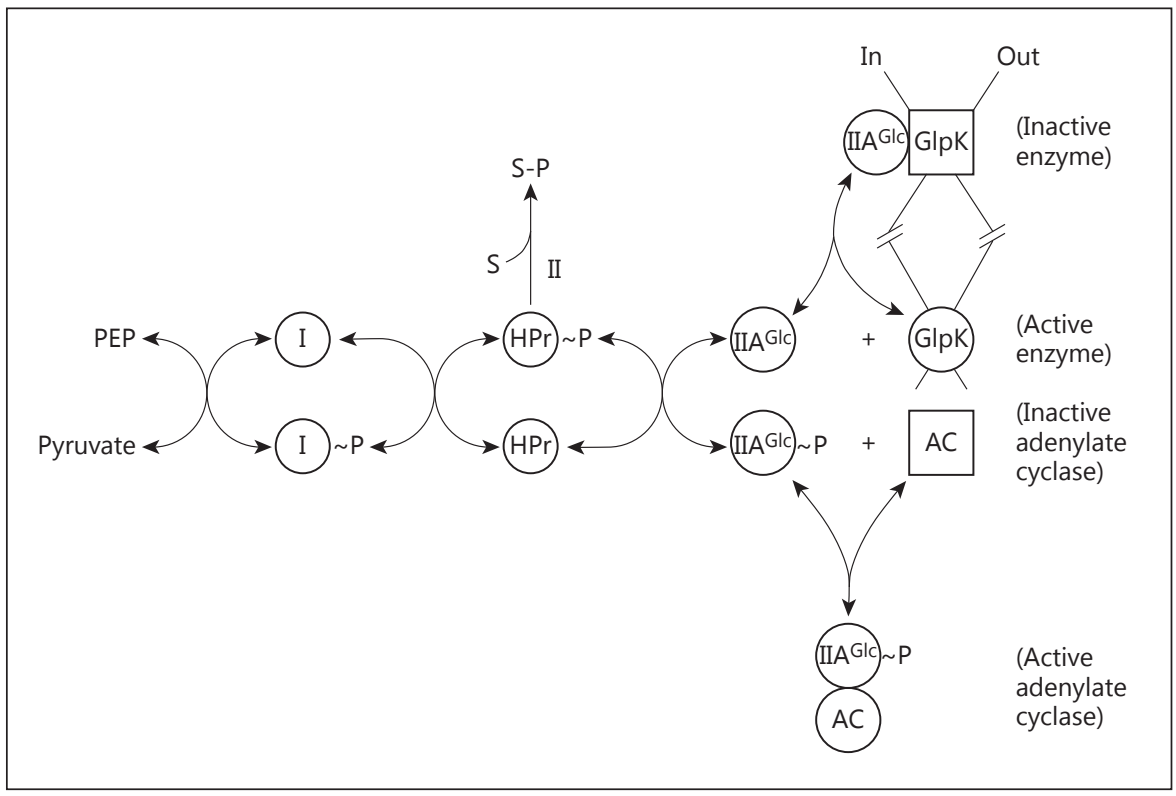

1997; Sondej et al., 1999]. Evidence is also available showing that substrate binding to at least some of these target proteins enhances their affinities for IIA ${ }^{\text {Glc }}$ [Osumi and Saier, 1982; Saier et al., 1983]. Although the PTS-mediated regulation of cyclic (c)AMP synthesis (catabolite repression) is not as well defined from a mechanistic standpoint, allosteric activation of adenylate cyclase by phospho-IIA Glc appears to be involved (fig. 1) [Park et al., 2006]. We now report that the general process of PTS-catalyzed protein phosphorylation-dephosphorylation is important to the regulation of transposon-mediated directed mutation, specifically, upstream of the $g l p F K$ operon, essential for the utilization of glycerol. This short review summarizes the first evidence that the PTS can influence the mutation rate in a directed fashion [Saier and Zhang, 2014].

\section{Darwin and Lamarck}

Charles Darwin is frequently considered to be the greatest biologist who ever lived [Trevors and Saier, 2011]. This is because he recognized and provided extensive evidence for the fact that all living organisms on Earth arose in an evolutionary process, accounting for their similarities, differences and relatedness (the Third Law of Biology) [Trevors and Saier, 2011]. However, 65 years before Darwin, the French naturalist, Jean Baptiste Pierre Antoine de Monet Chevalier de la Marck (Lamarck) had been a proponent of the idea that living or- ganisms arose in an evolutionary process according to natural laws. He developed the first theory of inheritance of acquired characteristics which became known as 'soft inheritance' or 'Lamarckism'. He believed in a 'complexifying force' driven by physiological need and the use (or disuse) of phenotypic characteristics [Burkhardt, 2013; Corsi, 2009]. In brief, he suggested that a giraffe acquired its long neck or an elephant acquired its long nose because it stretched and used the relevant organ for purposes that facilitated survival. Due to this proposal, which was very reasonable at his time, and perhaps at some level today as well, people ridiculed Lamarck as illustrated in figure 2.

\section{The Proposal of Directed Mutation}

John Cairns at Harvard University [Cairns et al., 1988] and Barry Hall at the University of Rochester [Hall, 1991] proposed and argued in favor of the concept of directed mutation. They suggested that organisms could respond to environmental stresses by reorganizing or changing their genes in a purposeful fashion. In other words, such mutations would occur with higher frequency if they relieved the stress that caused them. Thus, if a population is repeatedly subjected to cyclic and predictable environmental shifts, there can sometimes be selection for mutational mechanisms that increase the probability of mutational changes under certain well-defined physiological conditions. 


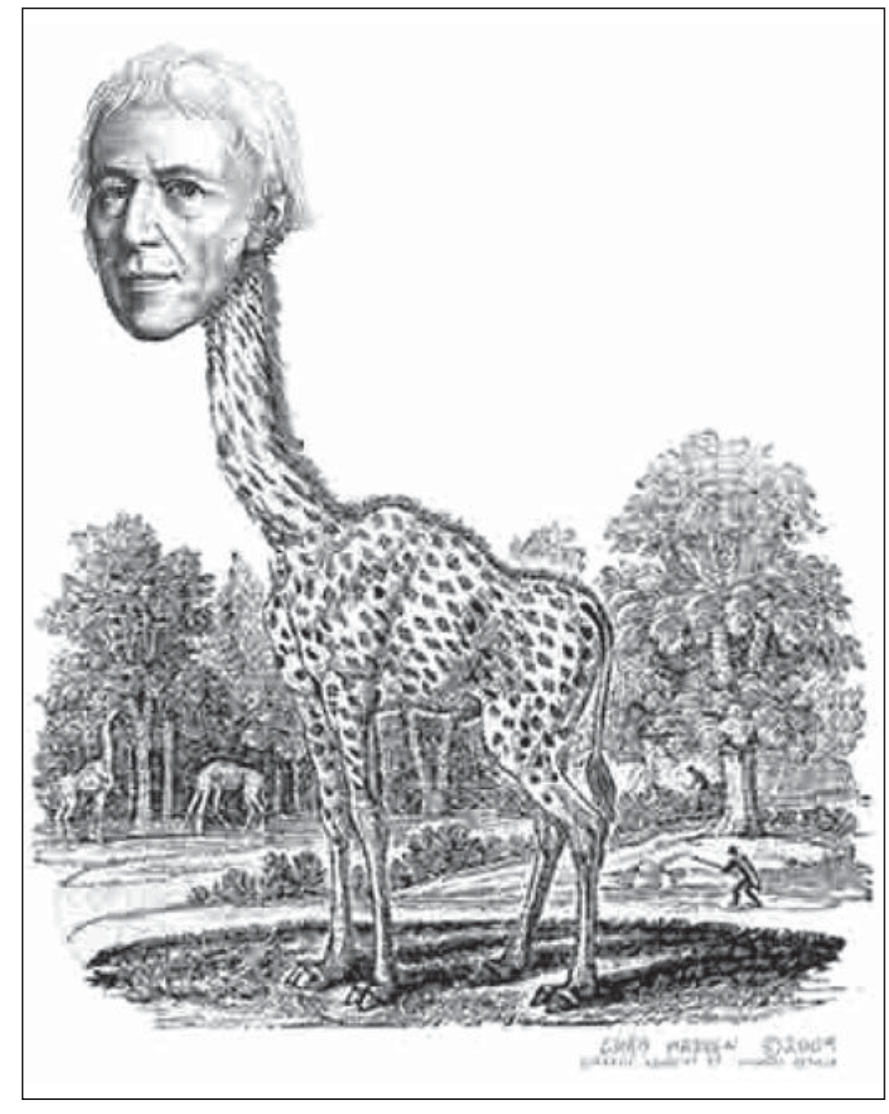

Fig. 2. Caricature of Lamarck, showing his face replacing that of a giraffe. It refers to the idea that because the giraffe repeatedly tried to stretch its neck to reach the leaves of a tree, its neck, and those of its offspring, became permanently longer. Such inheritance has been termed 'Lamarckian inheritance' and implies that evolutionary change is directed by need. Only the level at which or the mechanism by which this occurs distinguishes the proposals now attributed to Lamarck and Darwin. This figure was reproduced with permission by Chris Madden.

There is good evidence for such a mechanism as a result of microsatellite mutational analyses in some bacteria [Moxon and Wills, 1999]. However, evolution can take other directions; for example, the evolution of phenotypic plasticity may take more time and may not be advantageous in bacteria that have physiological limits to what they can do and still survive in a competitive situation [Field et al., 1999]. Contingency loci have acquired the ability to evolve rapidly if the environment changes in a predictable way [Moxon and Wills, 1999]. The evolution of highly specific hypermutation and recombination mechanisms in the adaptive immune system is another example of how the structure of the genome can evolve to meet challenges that vary in a predictable way [Kato et al., 2012; Keim et al., 2013; Orthwein and Di Noia, 2012].
If the proposal of directed mutation were to be verified, it could shift the course of evolution in a nonrandom and accelerated way. However, it goes against the dogma of our time, which states that mutations occur randomly and that the advantageous mutations are selected only after they arise, a concept that is in detail now known to be inaccurate [Caporale and Doyle, 2013; Galhardo et al., 2007]. The notion of directed mutation has been highly controversial and is not generally accepted in the scientific community, even today [Roth et al., 2006; Saier, 2011; Zhang and Saier, 2009a, 2011].

\section{Transposons}

Transposons are 'jumping genes', DNA elements that move autonomously to distal locations on a chromosome or plasmid. They were discovered in 1949 by Barbara McClintock while studying pigment variegation in corn seed kernels [Fedoroff, 2012; Ravindran, 2012]. These 'hopping' or transposition events gave rise to unstable mutations that occurred at high frequencies, much higher than normal mutation rates [Bennett, 2004]. Transposons have been identified in virtually all living organisms. Bacterial insertion sequence (IS) elements are the smallest transposons known [Siguier et al., 2006]. These small genomic elements have the potential to activate the expression of 'silent' operons, thus allowing the metabolism of compounds that otherwise could not be used as nutrients [Georgiev and Lambadjieva, 1981; Reynolds et al., 1986; Schnetz and Rak, 1992]. It is interesting to note that in humans over $30 \%$ of the chromosomal DNA is derived from (retro)transposons [Huang et al., 2012]. It seems likely that they serve useful purposes that are currently unrecognized.

How do IS elements function? They usually recognize and insert into specific target sites in the host DNA (fig. 3) [Craig, 1997]. The transposition event can be regulated by processes involving frameshifting, antisense RNA, or DNA methylation [Nagy and Chandler, 2004]. It can occur either by a replicative mechanism, in which case a new identical element, which 'hops', is synthesized, giving rise to an increase in its number by one, or by a nonreplicative mechanism, in which case the element is cleaved out of its original site and transferred elsewhere. Transposon hopping can inactivate a gene or activate the expression of an operon [Craig, 1997; Reynolds et al., 1981, 1986; Schnetz and Rak, 1992]. IS5, the transposon of interest in the studies described below, encodes a transposase, the enzyme that catalyzes the transposition event, the Ins5 A 


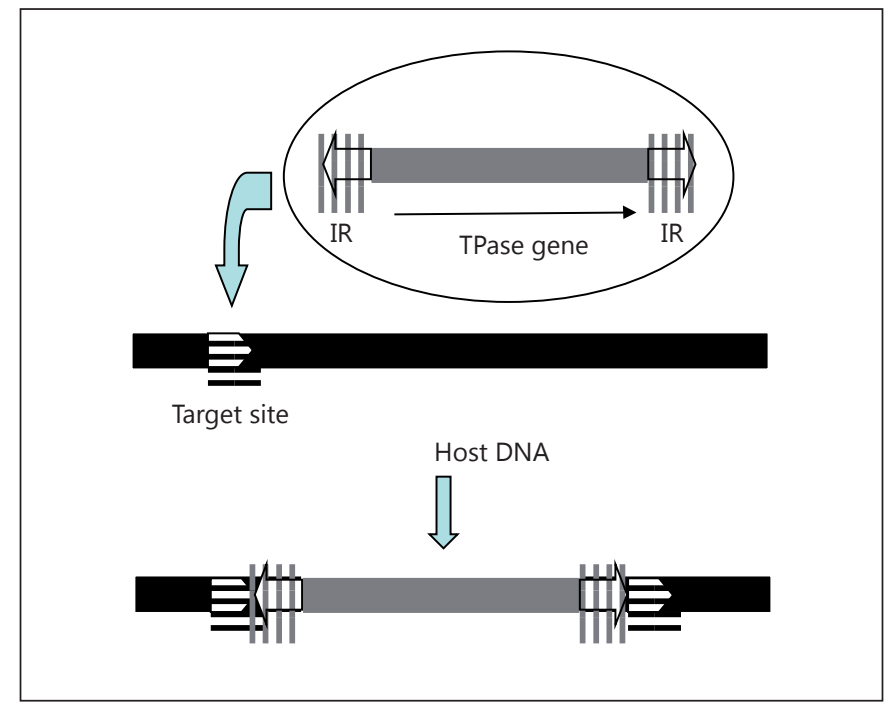

Fig. 3. Schematic depiction of a small transposon, a DNA IS element. It identifies a target site in the chromosome (indicated by the horizontal white bars) and inserts into that site while duplicating the target site. IR = Inverted repeat, represented by the vertical white bars; TPase $=$ the transposase which catalyzes the IS element transposition event.

protein (fig. 3) [Umenhoffer et al., 2010]. However, downstream of the Ins5 A structural gene in the DNA of IS5 are: (a) a binding site for the nucleoid protein, IHF, and (b) a permanent DNA bend (see fig. 3) [Zhang and Saier, 2009b]. These two sites, in combination, prove to be essential for IS5-mediated activation of the $g l p F K$ operon [Zhang and Saier, 2009b].

\section{The glp Regulon of E. coli}

The $g l p F K$ operon, one of five operons in the glycerol $(g l p)$ regulon, is essential for growth on glycerol. It encodes the glycerol facilitator (GlpF), which facilitates the uptake of glycerol from the medium, and glycerol kinase (GlpK), which phosphorylates glycerol with ATP to yield glycerol-3-phosphate, the inducer of the $g l p$ regulon [Holtman et al., 2001; Mao et al., 1999]. The $g l p D$ gene, encoding glycerol-3-phosphate dehydrogenase (GlpD), completes glycerol-specific metabolism under aerobic conditions, yielding dihydroxyacetone-phosphate that feeds directly into glycolysis [Fraser and Yamazaki, 1980; Ormo et al., 1998]. The glycerol repressor (GlpR), which controls expression of the five operons of the $g l p$ regulon, is encoded by a gene $(g l p R)$ that maps elsewhere on the $E$. coli chromosome.

Directed Mutation by the E. coli

Phosphoenolpyruvate:Sugar PTS

\section{The glpFK Operon}

The control region of the $g l p F K$ promoter region is shown in figure 4 . There are four binding sites (operators) for GlpR (O1-O4) and two for the cAMP receptor protein, Crp, CrpI and CrpII. The -35 hexanucleotide sequence of the promoter overlaps $\mathrm{O} 3$ and $\mathrm{CrpII}$, while the -10 promoter hexanucleotide sequence overlaps O4 (fig. 4) [Zhang and Saier, 2009a]. GlpR, in the free form, without glycerol-3-phosphate bound to it, binds its operators to repress $g l p F K$ operon expression, while Crp, in the cAMP-bound form, binds its two sites, Crp I and Crp II, to activate $g l p F K$ operon expression. Thus, GlpR negatively regulates $g l p F K$ expression, while Crp with bound cAMP positively regulates expression.

Due to the dependency of $g l p F K$ operon expression on the cAMP-Crp complex, $c r p^{-}$or $c y a^{-}$(adenylate cyclase) mutants cannot utilize glycerol. Wild-type cells that can make cAMP and have Crp can grow on glycerol, but not in the presence of a nonmetabolizable glucose analogue such as 2-deoxyglucose (2DG) or a-methylglucoside ( $a M G)$ which inhibits GlpK and Cya activities. The directed mutational event described below allows the bacterium to overcome the inhibitory effect of a nonmetabolizable sugar analogue.

\section{Evidence for Directed Mutation of the glpFK Operon}

When crp or cya mutant cells are plated on minimal glycerol plates, or when wild-type cells are plated on the same plates containing 2DG or aMG, IS5 insertion mutations arise after a lag period during which the cells are starving [Zhang and Saier, 2009a, 2014]. Starvation in the presence of glycerol activates the hopping of IS5 to the $g l p F K$-activating site, and insertion of IS5 relieves this stress [Zhang and Saier, 2009a]. How this activation occurs has been the subject of extensive studies [Saier and Zhang, 2014], and numerous control experiments have led to the conclusion that this activation event is specific for this one insertional site in front of the glpFK promoter [Zhang and Saier, 2009a, b]. This specificity could have been selected for by currently recognized mechanisms of Darwinian evolution.

By knocking out the $g l p R$ gene, encoding the glycerol repressor, it could be shown that this protein, when bound to the DNA, inhibits the activational insertion of IS 5 by about 10-fold in the absence of glycerol. Additionally, the presence of glycerol in the medium of wild-type cells allows the generation of glycerol-3-phosphate in the 
Fig. 4. The E. coli glpFK promoter region showing: (1) the ctaa insertion site (duplicated following IS5 insertion), (2) the four adjacent GlpR operators (binding sites), O1-O4, (3) the two adjacent Crp binding sites, CrpI and CrpII, (4) the -35 and -10 hexanucleotide regions of the promoter, (5) the transcriptional start site $(+1)$ and (6) the ribosome binding site (RBS) for initiation of translation of the first structural gene, the $g l p F$ gene within the $g l p F K$ operon. The start codon of the $g l p F$ gene (atg) and a downstream EcoRI restriction site are also shown.

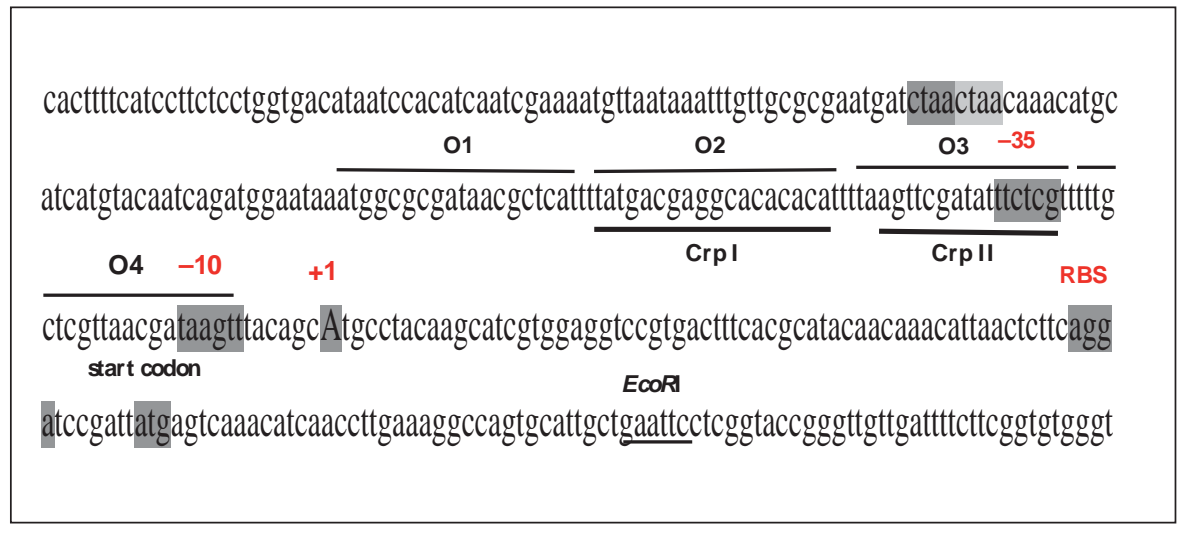

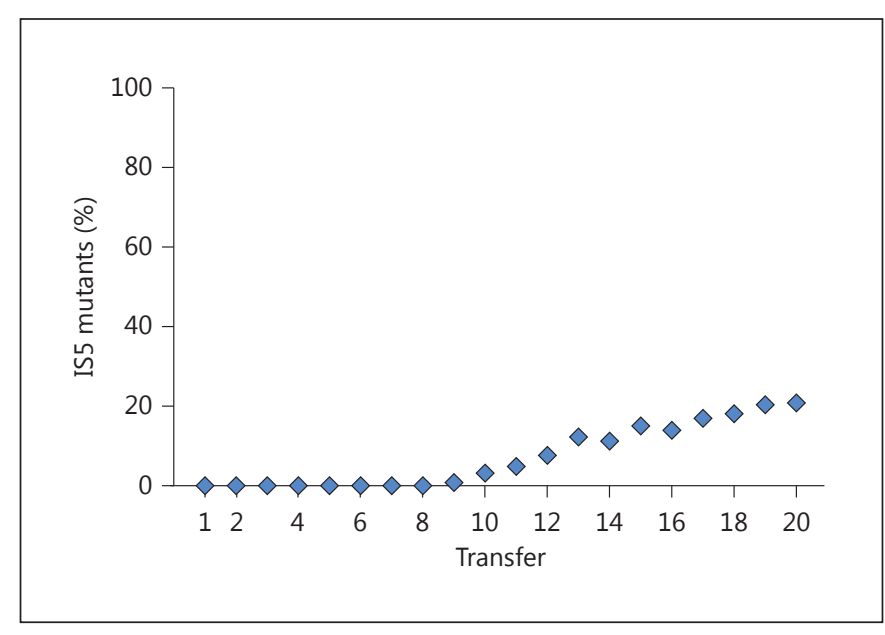

Fig. 5. Slow accumulation of IS5 insertion mutants over time when wild-type bacteria are incubated in minimal medium M9 in the presence of glycerol $(0.5 \%)$ and 2DG $(0.1 \%)$. A single transfer is equivalent to about 8 generations.

cytoplasm. This is responsible for a 10-fold increase in mutation rate, and this effect is mediated by GlpR. This conclusion was confirmed by the overexpression of $g l p R$, which greatly depressed the $g l p^{+}$mutation rate when glycerol was absent, but not when it was present [Zhang and Saier, 2009a]. Thus, in the presence of exogenous glycerol, cells possessing an intact $g l p R$ gene showed a high mutation rate, although this high rate was constitutively observed without glycerol in cells lacking GlpR. These results led to the conclusion that GlpR mediates the activating effect of glycerol on the insertion of IS5 into the $g l p F K$-activating site. It should be noted that wild-type cells evolved the GlpR-mediated mechanisms of directed mutations, and that the behavior of $g l p R$ and crp mutants (see below), while revealing the mechanistic details of the regulatory process, is not relevant to wildtype cell physiology. This behavior merely reflects the mechanisms of mutational control in the wild-type cells. In a population of $E$. coli cells in nature, $c r p$ and $g l p R$ mutants are not expected to be present because loss of Crp and/or GlpR decreases the ability of the cells to compete with wild-type cells under most conditions.

\section{Independent Involvement of GlpR in Transcriptional Regulation and Directed Mutation}

As noted above, cytoplasmic glycerol-3-phosphate activates transcription of the $g l p F K$ operon as well as IS5 insertion into the $g l p F K$-activating site, and these two effects are both mediated by GlpR. We considered the possibility that one was a consequence of the other. Since GlpR binds to four operators, O1-O4, in the glpFK control region, we wondered which of these play roles in the two events. Consequently, O1 (the upstream operator) and $\mathrm{O} 4$ (the downstream operator; see fig. 4) were separately mutated (by site-specific mutagenesis) and the consequent phenotypes were characterized. Mutating O1, so that this site could not bind GlpR, had little effect on transcriptional regulation of $g l p F K$ expression by GlpR, but mutating $\mathrm{O} 4$ gave rise to expressional activation in the absence of glycerol to a high constitutive level. Thus, $\mathrm{O} 4$ but not $\mathrm{O} 1$ primarily controls transcription [Zhang and Saier, 2009a]. By contrast, mutating O1 had a dramatic effect on the IS5-mediated mutation rate, although mutating O4 had a minimal effect [Zhang and Saier, 2009a]. It was therefore clear that the IS5-mediated mutation rate is independent of the $g l p F K$ expression level. The two processes are regulated independently of each other, even 
Fig. 6. Schematic depiction of the proposed regulation of $g l p F K$ operon transcription (indicated by the arrow in front of the $g l p F$ gene) and activation by IS5 insertion (upstream of the promoter, where the GlpR and the CAMP-Crp binding sites are. O1$\mathrm{O} 4$ are the four GlpR binding sites, and CrpI and CrpII are the two Crp binding sites. The four different possible outcomes, depending on conditions, are shown to the right of the vertical arrow. In the upper part of the figure, $\rightarrow$ represents activation and $\top$ represents inhibition or repression.

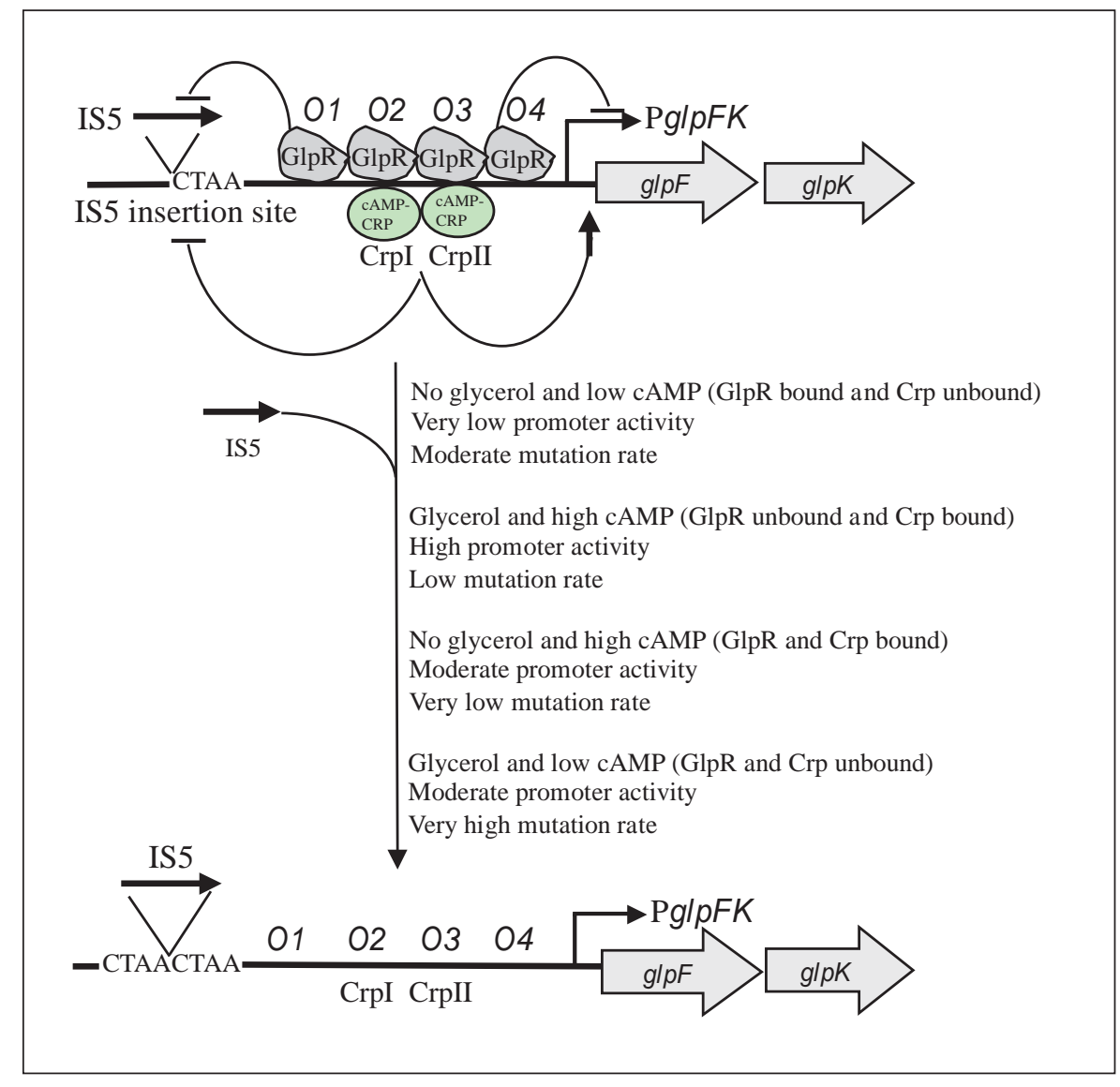

though both are controlled by GlpR. Possibly O1 evolved to control IS5-mediated $g l p F K$ activation while O4 evolved to control $g l p F K$ transcriptional expression in response to the availability of exogenous glycerol.

\section{Involvement of the CAMP-Crp Complex in Directed Mutation}

As noted above, IS5-mediated $g l p F K$-activating insertions could be demonstrated in either crp or cya mutants in a variety of liquid or solid media with highest rates occurring in the presence of glycerol, but with low to negligible concentrations of the cAMP-Crp complex. The behavior of the crp and cya mutants reflects the mechanism by which the hopping of IS5 in the glpFK-activating site is regulated by the cAMP-Crp complex. These mutants, because of their reduced growth rates, are not found in nature. The suppression of IS5 hopping to the activating site by the cAMP-Crp complex proved to depend on the two Crp binding sites in the $g l p F K$ control region (fig. 4).
Furthermore, it could be shown that glucose, which strongly inhibits this specific insertional event, did not suppress hopping of IS5 to the $g l p F K$-activating site by a mechanism dependent on the cAMP-Crp complex [Saier and Zhang, 2014]. Our studies clearly showed that glucose exerts its effect on IS5 insertion into the upstream glpFK site independently of Crp, Cya and GlpR. However, the mechanism of glucose control is not yet understood.

What were the conditions under which this directed mutational mechanism might have evolved in wild-type E. coli precursor cells? This question led to the possibility that it evolved to allow glycerol utilization when growth was inhibited by the presence of a bacteriostatic compound such as a sugar analogue (e.g. 2-deoxy-glucose; see fig. 5), that is known to inhibit both adenylate cyclase and glycerol kinase (see fig. 1) [Saier and Zhang, 2014]. Since the process of IS5 hopping is fully reversible [Zhang and Saier, 2011], this process could be of evolutionary significance. We suggest that the first GlpR binding site (O1) and the tetranucleotide IS5 insertion site upstream of the glpFK promoter evolved to allow control over this IS5 insertional event. 


\section{Concluding Remarks}

A model that provides an explanation for the findings summarized in this short review is shown in figure 6. Binding of either GlpR or the cAMP-Crp complex to the $g l p F K$ control region inhibits IS5 insertion into the upstream site, even though GlpR represses while the cAMPCrp complex activates transcription. It is not currently known if the inhibitory effects of GlpR and Crp binding to the DNA on IS5 insertion occurs by an allosteric mechanism involving competitive binding of these proteins with the transposase, or if a signal is transmitted through the DNA from the Crp and GlpR binding sites to the IS5 insertion site. Regardless, since the PTS directly regulates glycerol kinase (GlpK), which makes the inducer glycerol-3-phosphate, and adenylate cyclase (Cya), which makes cAMP (fig. 1), it seems likely that one of the regulatory roles of the PTS is to control IS5 insertion into the specific site upstream of the $g l p F K$ promoter that specifically enhances the strength of this promoter [Saier and Zhang, 2014]. Thus, the PTS indirectly regulates the directed mutational process that allows the bacteria to overcome the bacteriostatic effects of nonmetabolizable sugar analogues when E. coli is exposed to glycerol as a primary source of carbon and energy. Since nonmetabolizable sugar analogues are prevalent in nature [He et al., 2002; Kumar et al., 2013; Moller, 2010; Xi et al., 2014], this mechanism of directed mutation could have appeared in an evolutionary process. It is possible that transposonmediated directed mutation evolved by the introduction of a tetranucleotide IS targeting site as well as the O1 GlpR operator, upstream of the glpFK promoter. Alternatively, both the transposon and the regions of the genome which
IS5 targets could have coevolved in response to a predictably fluctuating environment. Thus, the probability of a beneficial mutation would be increased. Since directed mutation is of obvious benefit to the organism, it is reasonable to conclude that it evolved in response to natural selection.

An important condition for the evolution of this proposed mechanism of directed mutation is that the bacteria must have repeatedly faced a situation in the past in which glycerol utilization is blocked by the presence of nonmetabolizable PTS sugar analogues. It is intriguing that this process of acquiring phenotypic plasticity allows an E. coli population to make this switch as soon as the environment changes. Perhaps this is because the metabolic cost of maintaining such a capability is minimal compared to the price of entire population extinction. Bacteria can survive catastrophic environmental changes that kill most of the cells in the population much more readily than complex higher organisms can. They may be able to survive the cost of waiting for a directed mutation to happen. We thus recognize the potential for the synthesis of ideas attributed to Lamarck and Darwin, although directed mutation, a concept consistent with the Lamarckian postulates, can be considered to be the result of straightforward Darwinian selection.

\section{Acknowledgements}

This work was supported by NIH grant GM077402. We thank Fengyi Tang and Joshua Asiaban for assistance in the preparation of this manuscript, and Josef Deutscher, Bernhard Erni, Joseph Lengeler and Chris Wills for useful suggestions for the improvement of this paper.

\section{References}

Barabote RD, Saier MH Jr: Comparative genomic analyses of the bacterial phosphotransferase system. Microbiol Mol Biol Rev 2005;69:608634.

Bennett PM: Genome plasticity: insertion sequence elements, transposons and integrons, and DNA rearrangement. Methods Mol Biol 2004;266:71-113.

Bluschke B, Volkmer-Engert R, Schneider E: Topography of the surface of the signal-transducing protein EIIA ${ }^{\text {Glc }}$ that interacts with the MalK subunits of the maltose ATP-binding cassette transporter $\left(\mathrm{MalFGK}_{2}\right)$ of Salmonella typhimurium. J Biol Chem 2006;281:12833 12840 .
Burkhardt RW Jr: Lamarck, evolution, and the inheritance of acquired characters. Genetics 2013;194:793-805.

Cairns J, Overbaugh J, Miller S: The origin of mutants. Nature 1988;335:142-145.

Caporale LH, Doyle J: In Darwinian evolution, feedback from natural selection leads to biased mutations. Ann NY Acad Sci 2013;1305: 18-28.

Corsi P: Evolution pioneers: Lamarck's reputation saved by his zoology. Nature 2009;461:167.

Craig NL: Target site selection in transposition. Annu Rev Biochem 1997;66:437-474.

Fedoroff NV: Mcclintock's challenge in the 21st century. Proc Natl Acad Sci USA 2012;109: 20200-20203.
Field D, Magnasco MO, Moxon ER, Metzgar D, Tanaka MM, Wills C, Thaler DS: Contingency loci, mutator alleles, and their interactions: synergistic strategies for microbial evolution and adaptation in pathogenesis. Ann NY Acad Sci 1999;870:378-382.

Fraser AD, Yamazaki H: Characterization of an Escherichia coli mutant which utilizes glycerol in the absence of cyclic adenosine monophosphate. Can J Microbiol 1980;26:393-396.

Galhardo RS, Hastings PJ, Rosenberg SM: Mutation as a stress response and the regulation of evolvability. Crit Rev Biochem Mol Biol 2007; 42:399-435. 
Georgiev VP, Lambadjieva ND: Interactions between cholinergic drugs, $\gamma$-aminobutyric acid and cyclic guanosine monophosphate on picrotoxin-induced convulsive-seizure threshold. Neuropharmacology 1981;20:813-817.

Hall BG: Is the occurrence of some spontaneous mutations directed by environmental challenges? New Biol 1991;3:729-733.

He XM, Liu HW: Formation of unusual sugars: mechanistic studies and biosynthetic applications. Annu Rev Biochem 2002;71:701-754.

-Hoischen C, Levin J, Pitaknarongphorn S, Reizer J, Saier MH Jr: Involvement of the central loop of the lactose permease of Escherichia coli in its allosteric regulation by the glucose-specific enzyme IIA of the phosphoenolpyruvate-dependent phosphotransferase system. J Bacteriol 1996;178:6082-6086.

-Holtman CK, Pawlyk AC, Meadow ND, Pettigrew DW: Reverse genetics of Escherichia coli glycerol kinase allosteric regulation and glucose control of glycerol utilization in vivo. J Bacteriol 2001;183:3336-3344.

-Huang CR, Burns KH, Boeke JD: Active transposition in genomes. Annu Rev Genet 2012;46: 651-675.

-Kato L, Stanlie A, Begum NA, Kobayashi M, Aida $\mathrm{M}$, Honjo T: An evolutionary view of the mechanism for immune and genome diversity. J Immunol 2012;188:3559-3566.

-Keim C, Kazadi D, Rothschild G, Basu U: Regulation of aid, the B-cell genome mutator. Genes Dev 2013;27:1-17.

Kumar C, Yadav K, Archana G, Naresh Kumar G: 2-ketogluconic acid secretion by incorporation of Pseudomonas putida KT 2440 gluconate dehydrogenase (gad) operon in Enterobacter asburiae PSI3 improves mineral phosphate solubilization Curr Microbiol 2013;67:388-394.

Mao C, Ozer Z, Zhou M, Uckun FM: X-Ray structure of glycerol kinase complexed with an ATP analog implies a novel mechanism for the ATP-dependent glycerol phosphorylation by glycerol kinase. Biochem Biophys Res Commun 1999;259:640-644.

Møller BL: Functional diversification of cyanogenic glucosides. Curr Opin Plant Biol 2010; 13:338-347.

Moxon ER, Wills C: DNA microsatellites: agents of evolution? Sci Am 1999;280:94-99.

- Nagy Z, Chandler M: Regulation of transposition in bacteria. Res Microbiol 2004;155:387-98.

-Ormö M, Bystrom CE, Remington SJ: Crystal structure of a complex of Escherichia coli glycerol kinase and an allosteric effector fructose 1,6-bisphosphate. Biochemistry 1998;37: 16565-16572.
Orthwein A, Di Noia JM: Activation induced deaminase: how much and where? Semin Immunol 2012;24:246-254.

Osumi T, Saier MH Jr: Regulation of lactose permease activity by the phosphoenolpyruvate:sugar phosphotransferase system: evidence for direct binding of the glucose-specific Enzyme III to the lactose permease. Proc Natl Acad Sci USA 1982;79:1457-1461.

Park YH, Lee BR, Seok YJ, Peterkofsky A: In vitro reconstitution of catabolite repression in Escherichia coli. J Biol Chem 2006;281:64486454.

Postma PW, Lengeler JW, Jacobson GR: Phosphoenolpyruvate:carbohydrate phosphotransferase systems of bacteria. Microbiol Rev 1993; 57:543-594.

Ravindran S: Barbara McClintock and the discovery of jumping genes. Proc Natl Acad Sci USA 2012;109:20198-20199.

Reynolds AE, Felton J, Wright A: Insertion of DNA activates the cryptic bgl operon in E. coli k12. Nature 1981;293:625-629.

Reynolds AE, Mahadevan S, LeGrice SF, Wright A: Enhancement of bacterial gene expression by insertion elements or by mutation in a CAP-cAMP binding site. J Mol Biol 1986;191: 85-95.

Roth JR, Kugelberg E, Reams AB, Kofoid E, Andersson DI: Origin of mutations under selection: the adaptive mutation controversy. Annu Rev Microbiol 2006;60:477-501.

- Saier MH Jr: Protein phosphorylation and allosteric control of inducer exclusion and catabolite repression by the bacterial phosphoenolpyruvate:sugar phosphotransferase system. Microbiol Rev 1989;53:109-120.

- Saier MH Jr: Regulatory interactions involving the proteins of the phosphotransferase system in enteric bacteria. J Cell Biochem 1993;51: 62-68.

Saier MH Jr: Did adaptive and directed mutation evolve to accelerate stress-induced evolutionary change? J Mol Microbiol Biotechnol 2011; 21:5-7.

- Saier MH Jr, Novotny MJ, Comeau-Fuhrman D, Osumi T, Desai JD: Cooperative binding of the sugar substrates and allosteric regulatory protein (Enzyme IIIGlc of the phosphotransferase system) to the lactose and melibiose permeases in Escherichia coli and Salmonella typhimurium. J Bacteriol 1983;155:13511357.

Saier MH Jr, Roseman S: Sugar transport. inducer exclusion and regulation of the melibiose, maltose, glycerol, and lactose transport systems by the phosphoenolpyruvate:sugar phosphotransferase system. J Biol Chem 1976;251:6606-6615.
Saier MH Jr, Straud H, Massman LS, Judice JJ, Newman MJ, Feucht BU: Permease-specific mutations in Salmonella typhimurium and Escherichia coli that release the glycerol, maltose, melibiose, and lactose transport systems from regulation by the phosphoenolpyruvate:sugar phosphotransferase system. J Bacteriol 1978;133:1358-1367.

- Saier MH Jr, Zhang Z: Transposon-mediated directed mutation controlled by DNA binding proteins in Escherichia coli. Front Microbiol 2014;5:390.

Schnetz K, Rak B: IS5: a mobile enhancer of transcription in Escherichia coli. Proc Natl Acad Sci USA 1992;89:1244-1248.

-Seok YJ, Sun J, Kaback HR, Peterkofsky A: Topology of allosteric regulation of lactose permease. Proc Natl Acad Sci USA 1997;94:1351513519.

-Siguier P, Filee J, Chandler M: Insertion sequences in prokaryotic genomes. Curr Opin Microbiol 2006;9:526-531.

- Sondej M, Sun J, Seok YJ, Kaback HR, Peterkofsky A: Deduction of consensus binding sequences on proteins that bind IIA Glc of the phosphoenolpyruvate:sugar phosphotransferase system by cysteine scanning mutagenesis of Escherichia coli lactose permease. Proc Natl Acad Sci USA 1999;96:3525-3530.

Trevors JT, Saier MH Jr: Thermodynamic perspectives on genetic instructions, the laws of biology and diseased states. CR Biol 2011;334: $1-5$

Umenhoffer K, Feher T, Baliko G, Ayaydin F, Posfai J, Blattner FR, Posfai G: Reduced evolvability of Escherichia coli MDS42, an IS-less cellular chassis for molecular and synthetic biology applications. Microb Cell Fact 2010;9: 38.

Xi FM, Li CT, Mi JL, Wu ZJ, Chen WS: Three new olean-type triterpenoid saponins from aerial parts of Eclipta prostrata (L.). Nat Prod Res 2014;28:35-40.

Zhang Z, Saier MH Jr: A mechanism of transposon-mediated directed mutation. Mol Microbiol 2009a; 74:29-43.

Zhang Z, Saier MH Jr: A novel mechanism of transposon-mediated gene activation. PLoS Genet. 2009b. doi: 10.1371/journal.pgen. 1000689. Epub ahead of print.

-Zhang Z, Saier MH Jr: Transposon-mediated adaptive and directed mutations and their potential evolutionary benefits. J Mol Microbiol Biotechnol 2011;21:59-70.
Directed Mutation by the E. coli Phosphoenolpyruvate:Sugar PTS
J Mol Microbiol Biotechnol 2015;25:226-233 DOI: $10.1159 / 000375375$ 